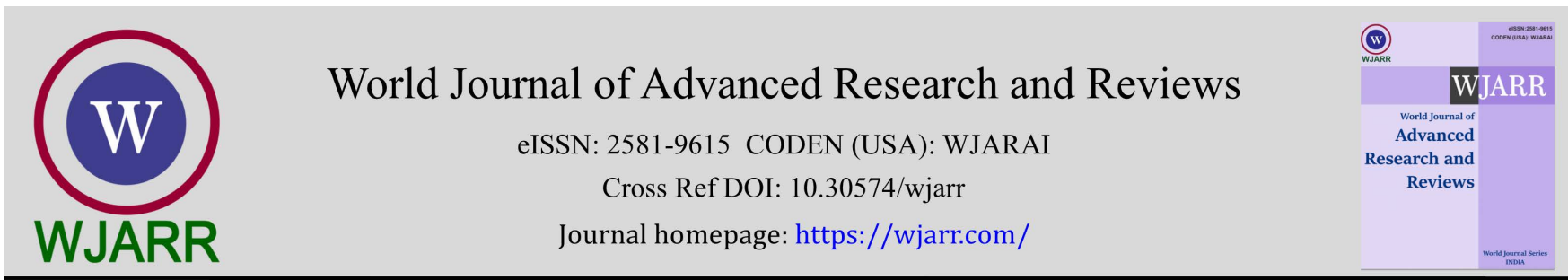

(RESEARCH ARTICLE)

\title{
Vehicle yaw rate simulation and control based on single-track model
}

\author{
Abdussalam Ali Ahmed ${ }^{1,}{ }^{*}$, Faraj Ahmed Elzarook Barood ${ }^{1}$ and Munir S. Khalifa ${ }^{2}$ \\ ${ }^{1}$ Mechanical and Industrial Engineering Department, Bani Waleed University, Bani Waleed/Libya \\ ${ }^{2}$ Computer \& IT Department, College of Electronic Technology, Bani Walid, Libya
}

World Journal of Advanced Research and Reviews, 2021, 10(01), 019-029

Publication history: Received on 23 February 2021; revised on 27 March 2021; accepted on 30 March 2021

Article DOI: https://doi.org/10.30574/wjarr.2021.10.1.0127

\begin{abstract}
When designing a vehicle, the most important variable that should be taken into account is the vehicle yaw rate, it represents an important indication of the vehicle's stability and control. This paper aims to demonstrate how to simulate and control the yaw rate of a vehicle using two control methods, the first is the Linear Quadratic control method (LQR) and the other one is neural network control. The classical single-track model is prominently used for yaw stability control analysis. One driving conditions performed is the steering input; the steering input in this work is set as step steering angle and a lane change manoeuvre. Simulation results showed that both control methods used produced good and convergent performance results for the vehicle under different driving conditions.
\end{abstract}

Keywords: Vehicle Stability; Single-Track Model; Yaw Rate; LQR; Neural Network Control.

\section{Introduction}

As vehicles are becoming more and more performing throughout the years, it turns out that their design process requires better knowledge of their behavior. One of the ways to get to that knowledge is to use mathematical models, which describe the behavior of the vehicle when given relevant parameters. Knowing these parameters is essential to run the models, and get the expected results. This study will focus on a simple vehicle description, known as "single track model" also referred to as "bicycle model". This model contains several parameters influencing its behavior.

In vehicle dynamic studies, the classical single-track model as shown in Figure 1 is prominently used for yaw stability control analysis [1].

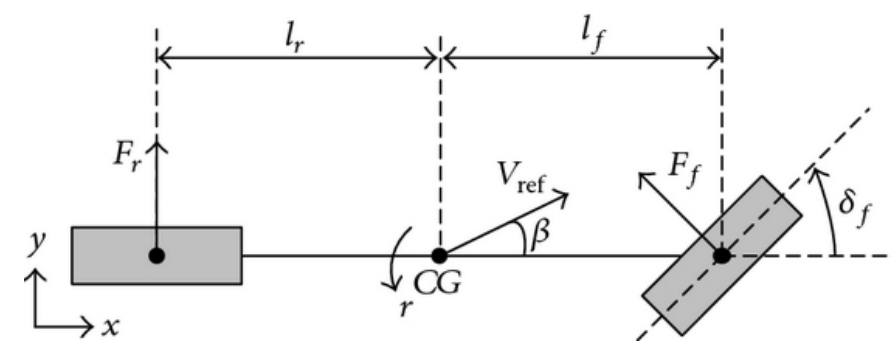

Figure 1 Single track model (bicycle model).

\footnotetext{
* Corresponding author: Abdussalam Ali Ahmed

Mechanical and Industrial Engineering Department, Bani Waleed University, Bani Waleed/Libya. 
In the single-track model the Left and right wheels at the front and rear axle are placed in a single wheel at the centerline of the vehicle.

In order to design an effective control system, it is necessary to determine an appropriate element of yaw stability control system [2]. In this work, the elements of yaw stability control system, that is, vehicle dynamic models, control objectives, active chassis control, and its control strategies as illustrated in Figure 2.

There are many automatic control methods that are used in the fields of life in general and in the automotive industry in particular [4].

There are several previous studies in which the simple track model of the vehicle was used in different control strategies [5], [6], [7]. In this paper, only two control techniques used which are LQR vehicle lateral stability control and neural network control.

In addition, to examine, and design the controller for yaw stability control system, vehicle dynamics models are essential where the mathematical modeling is obtained.

In general, there are two categories of vehicle dynamic model [2] that is nonlinear vehicle model and linearized vehicle model as painted in Figure 3.

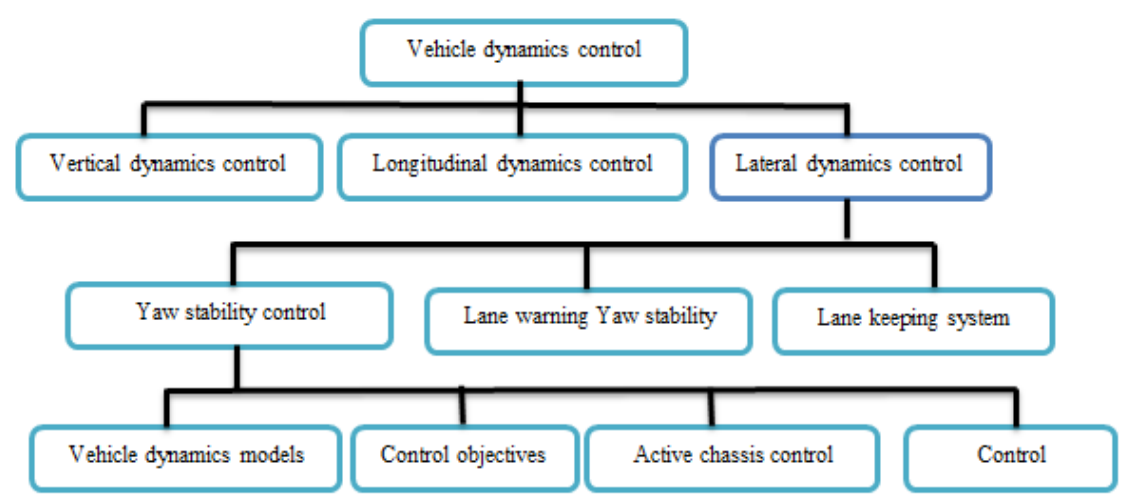

Figure 2 Yaw stability control system for vehicle lateral dynamic.

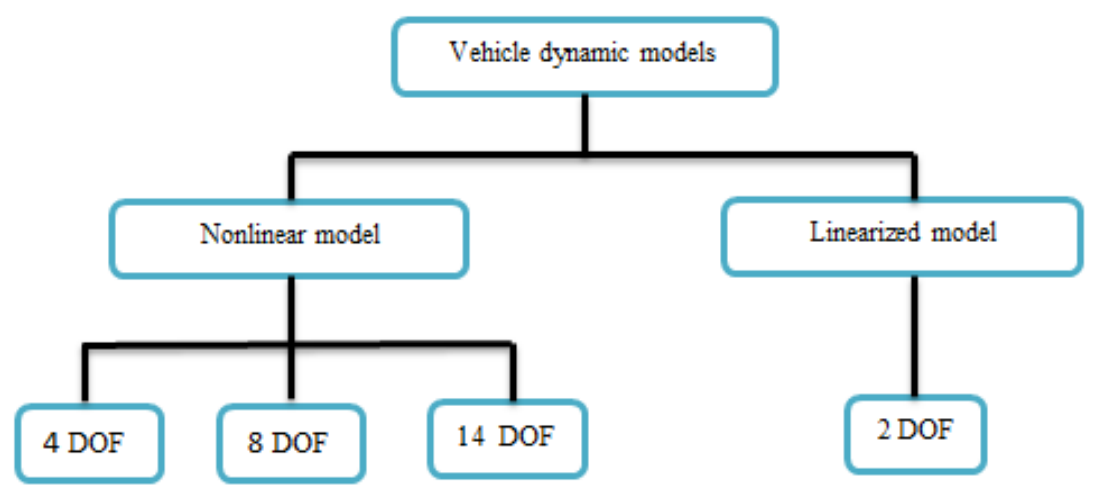

Figure 3 Vehicle dynamic model.

\section{Single Track Model}

The classical single-track model is the simplest model that accurately models the lateral dynamics of a road vehicle. It is valid for non-extreme situations; almost constant longitudinal speed and small side slip angle values. The two tires on the same axle are lumped together, resulting in one front and one rear tire. 


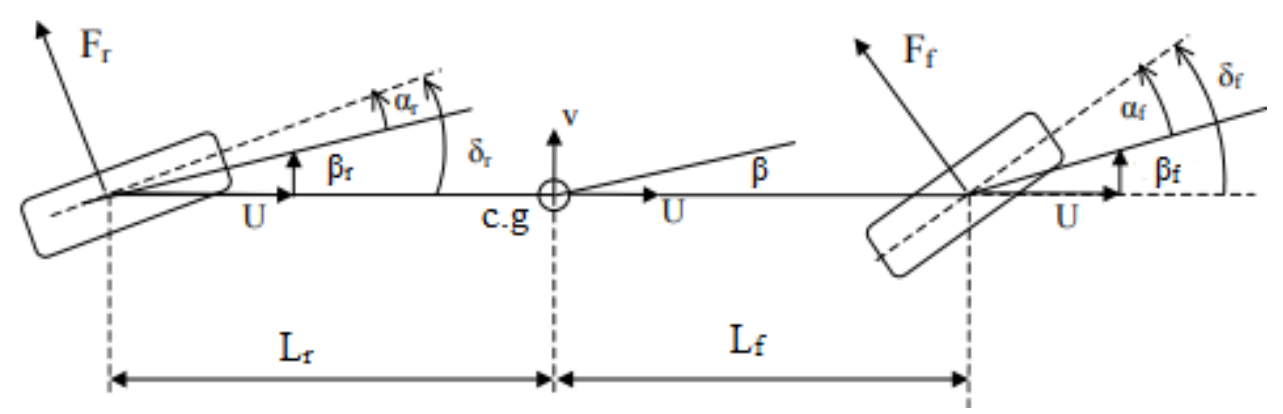

Figure 4 Free body diagram of the single-track model.

Table 1 Nomenclature used.

\begin{tabular}{|c|l|c|l|}
\hline c.g. & Center of gravity & $\boldsymbol{x}_{\boldsymbol{o}}, \boldsymbol{y}_{\boldsymbol{o}}$ & Inertial frame of reference \\
\hline $\mathrm{V}$ & Vehicle velocity at c.g. & $x-y$ & Chassis fixed frame of reference \\
\hline$\beta$ & Vehicle side slip angle & $V_{f}$ & Velocity at center of front tire \\
\hline$l_{f}$ & Distance between front axle and c.g & $V_{r}$ & Velocity at center of rear tire \\
\hline$l_{r}$ & Distance between rear axle and c.g & $\alpha_{f}$ & Front tire side slip angle \\
\hline$F_{f}$ & Front tire lateral force & $\alpha_{r}$ & Rear tire side slip angle \\
\hline$F_{r}$ & Rear tire lateral force & $\beta_{f}$ & Front tire velocity angle with x axis \\
\hline$\delta_{f}$ & Front tire steering angle & $\beta_{r}$ & Rear tire velocity angle with x axis \\
\hline$\delta_{r}$ & Rear tire steering angle & $\psi$ & Yaw angle \\
\hline $\mathrm{r}$ & yaw rate & & \\
\hline
\end{tabular}

To complete this work, Matlab Simulink model of single-track model should be completed as shown in figure 5, this model includes Tire Model, Vehicle Dynamics, Steering Angle Projection, Kinematics/Geometry, Simple Driver Model, and Trajectory Calculations.

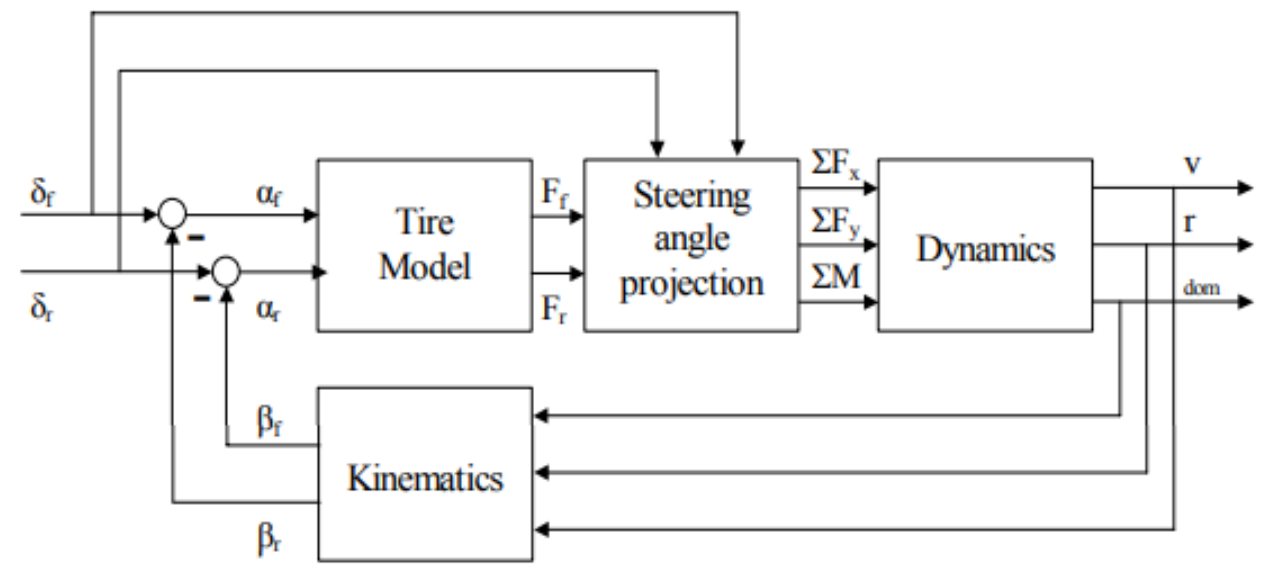

Figure 5 Block diagram of vehicle steering.

\subsection{Non-linear track model}

Nonlinear Model Equations: 


\subsubsection{Tire Model}

Linear tire model is used in this case:

$$
\begin{aligned}
& F_{f}=c f o \times\left(\delta_{f}-\beta_{f}\right)=c f o \times \alpha_{f} \\
& F_{r}=c f o \times\left(\delta_{r}-\beta_{r}\right)=c f o \times \alpha_{r}
\end{aligned}
$$

2.1.2. Vehicle Dynamics

$$
\begin{gathered}
m v(\dot{\beta}+\dot{\psi})=-F_{x} \sin \beta+F_{y} \cos \beta \\
m \dot{v}=F_{x} \cos \beta+F_{y} \sin \beta \\
J \ddot{\psi}=M_{z}
\end{gathered}
$$

\subsubsection{Steering Angle Projection}

$$
\begin{gathered}
\sum F_{x}=-F_{f} \sin \delta_{f}-F_{r} \sin \delta_{r} \\
\sum F_{y}=F_{f} \cos \delta_{f}+F_{r} \cos \delta_{r} \\
\sum M_{z}=l_{f} F_{f} \cos \delta_{f}-l_{r} F_{r} \cos \delta_{r}
\end{gathered}
$$

2.1.4. Kinematics/Geometry

$$
\begin{aligned}
& \beta_{f}=\tan ^{-1}\left(\tan \beta+\frac{\dot{\psi} l_{f}}{v \cos \beta}\right) \\
& \beta_{r}=\tan ^{-1}\left(\tan \beta-\frac{\dot{\psi} l_{r}}{v \cos \beta}\right)
\end{aligned}
$$

\subsubsection{Simple Driver Model}

A simple driver model was added to keep the longitudinal speed constant. The simplest PI cruise controller was used.

$$
\sum F_{x} \rightarrow \sum F_{x}+G_{P I}(s)\left(v_{\text {desired }}-v_{\text {measured }}\right)
$$

\subsubsection{Trajectory Calculations}

Distance traveled in the $\mathrm{x}_{0}$ and $\mathrm{y}_{0}$ directions were calculated using following equations;

$$
\begin{aligned}
& X=\int_{0}^{t} v \cos (\beta+\psi) d t \\
& Y=\int_{0}^{t} v \sin (\beta+\psi) d t
\end{aligned}
$$




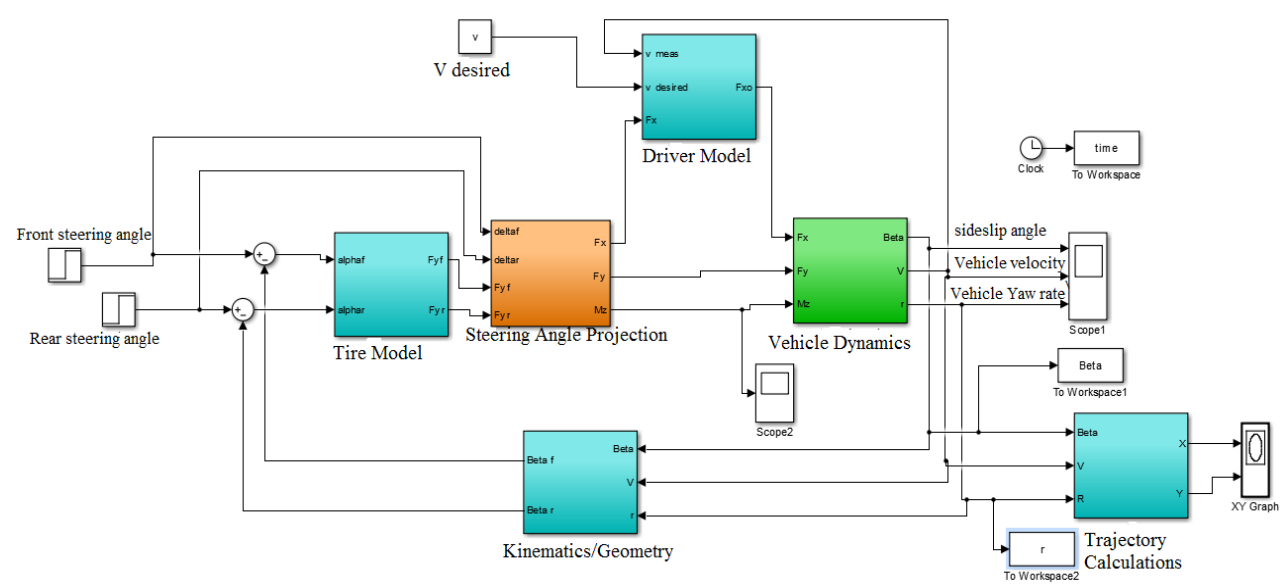

Figure 6 Simulink model of the nonlinear single-track model.

\subsection{Linear track model}

In vehicle dynamic studies, the reference vehicle model as shown in Figure 7 is prominently used for yaw stability control analysis and controller design.

The Linear Single Track Model contains the following subsystems:

Tire Model

Vehicle Dynamics

Force/Moment Coordinate Transformation (or Steering Angle Projection)

Kinematics/Geometry

Trajectory Calculations

\subsubsection{Tire Model}

Linear tire model was used.

$$
\begin{aligned}
& F_{f}=c f o \times\left(\delta_{f}-\beta_{f}\right)=c f o \times \alpha_{f} \\
& F_{r}=c f o \times\left(\delta_{r}-\beta_{r}\right)=c f o \times \alpha_{r}
\end{aligned}
$$

\subsubsection{Vehicle Dynamics}

Original nonlinear vehicle dynamics was

$$
\begin{gathered}
m v(\dot{\beta}+\dot{\psi})=-F_{x} \sin \beta+F_{y} \cos \beta \\
m \dot{v}=F_{x} \cos \beta+F_{y} \sin \beta \\
J \ddot{\psi}=M_{z}
\end{gathered}
$$

The force moment coordinate transformation equations will become as follows with small beta and constant velocity assumptions:

$$
\begin{gathered}
m v(\dot{\beta}+\dot{\psi})=F_{y} \\
J \ddot{\psi}=M_{z}
\end{gathered}
$$




\subsubsection{Force / Moment Coordinate Transformation}

Original nonlinear steering angle projection was

$$
\begin{gathered}
\sum F_{x}=-F_{f} \sin \delta_{f}-F_{r} \sin \delta_{r} \\
\sum F_{y}=F_{f} \cos \delta_{f}+F_{r} \cos \delta_{r} \\
\sum M_{z}=l_{f} F_{f} \cos \delta_{f}-l_{r} F_{r} \cos \delta_{r}
\end{gathered}
$$

The force moment coordinate transformation equations will become as follows with small steering angle assumptions:

$$
\begin{gathered}
\sum F_{y}=F_{f}+F_{r} \\
\sum M_{z}=l_{f} F_{f}-l_{r} F_{r}
\end{gathered}
$$

\subsubsection{Kinematics Geometry}

Original kinematics/geometry relation was

$$
\begin{aligned}
& \beta_{f}=\tan ^{-1}\left(\tan \beta+\frac{\dot{\psi} l_{f}}{v \cos \beta}\right) \\
& \beta_{r}=\tan ^{-1}\left(\tan \beta-\frac{\dot{\psi} l_{r}}{v \cos \beta}\right)
\end{aligned}
$$

The kinematics/geometry relation equations will become as follows with small chassis front and rear slip angles assumptions:

$$
\begin{aligned}
& \beta_{f}=\left(\beta+\frac{\dot{\psi} l_{f}}{v}\right) \\
& \beta_{r}=\left(\beta-\frac{\dot{\psi} l_{r}}{v}\right)
\end{aligned}
$$

\subsubsection{Trajectory Calculations}

In order to investigate about the instantaneous position of the vehicle;

$$
\begin{aligned}
& X=\int_{0}^{t} v \cos (\beta+\psi) d t \\
& Y=\int_{0}^{t} v \sin (\beta+\psi) d t
\end{aligned}
$$

Figures (5-7) show the Simulink model of the linear single-track model, vehicle yaw rate, and the vehicle body sideslip angle. 


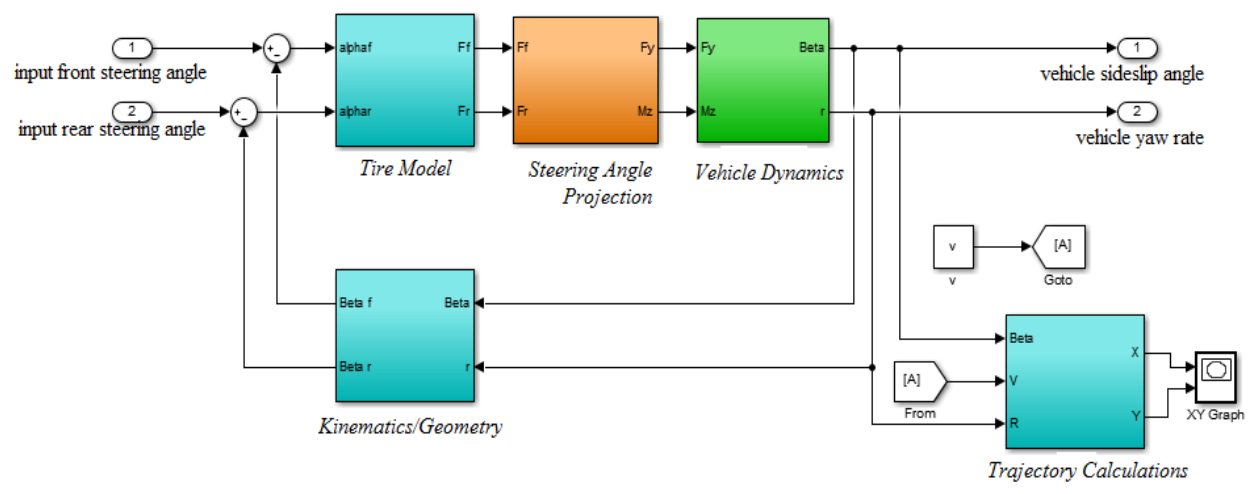

Figure 7 Simulink model of the linear single-track model

In this paper, linear single-track model yaw rate and has been taken as a desired yaw rate at different steering conditions as shown in figures 8 and 9, the steering input is set as step steering angle and a lane change manoeuvre.

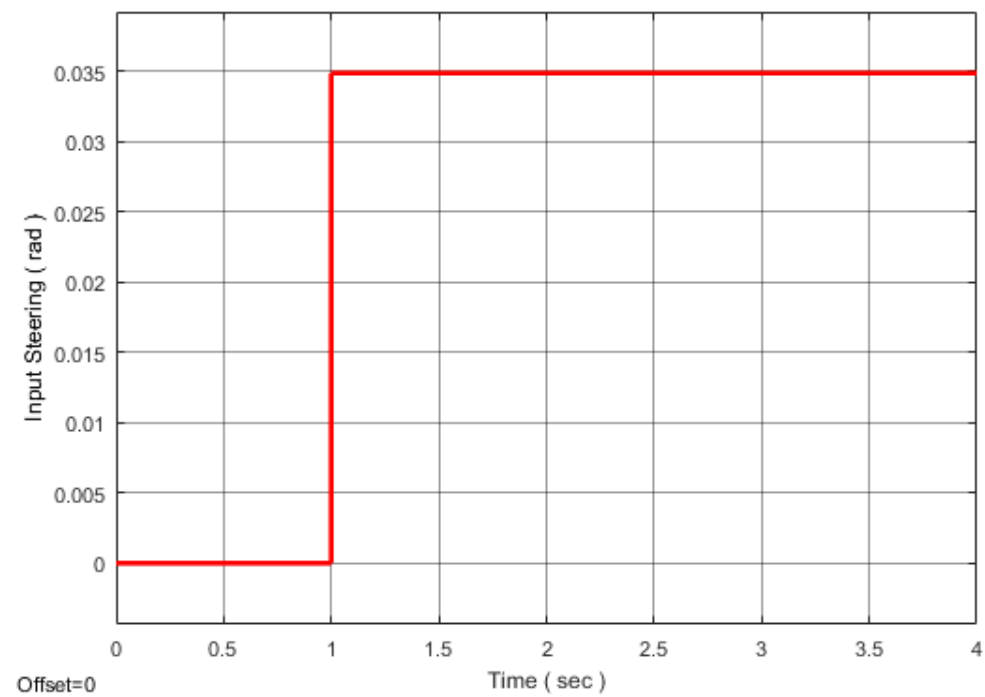

Figure 8 The steering input of vehicle with a step angle.

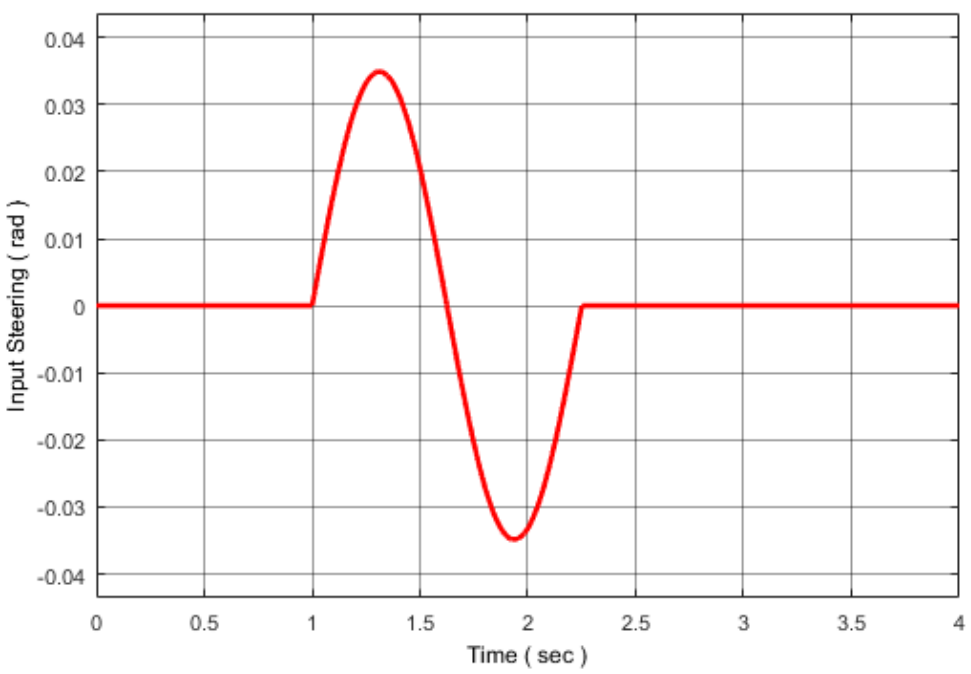

Figure 9 The steering input of vehicle with a lane-change maneuverer. 


\section{Control strategy}

\subsection{Neural Network Controller}

Artificial neural networks are forecasting methods that are based on simple mathematical models of the brain. They allow complex nonlinear relationships between the response variable and its predictors. A neural network can be thought of as a network of "neurons" which are organized in layers. The predictors (or inputs) form the bottom layer, and the forecasts (or outputs) form the top layer. There may also be intermediate layers containing "hidden neurons".

In this part, the controller structure uses a neural network with two input signals, which are the plant output and the reference signal, whereas the output signal is delayed control as illustrated in the figure below.

This network works with supervised learning where a data set is provided to train the network system before simulation runs. Figure 10 shows the implemented suspension system includes a neural network controller; this controller block is available in the SIMULINK library.

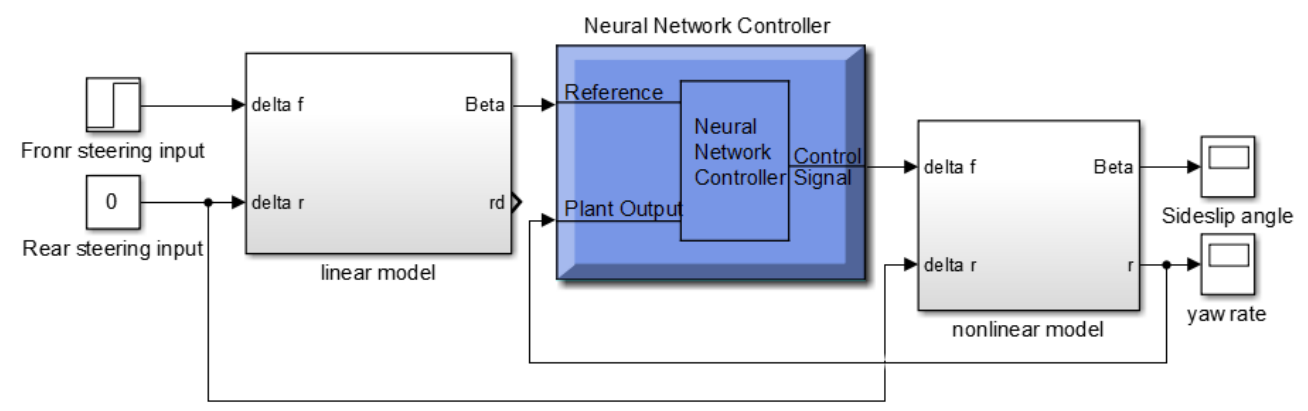

Figure 10 Simulink model for active suspension system with Neural Network Controller.

\subsection{LQR Vehicle lateral stability control}

The LQR approach to control the vehicle is widely used in the background of many studies in the field of the vehicle dynamics. It was used in a simple quarter car model, a half car model, as well as in a full vehicle model. The strength of the LQR method is that when using the performance factor index that can be weighted according to the engineers' desires and aspirations or other limitations. With this approach type, an optimal result can be achieved when factors of the performance index are taken into account.

The LQR method for the vehicle dynamics has been suggested and proposed to improve passenger comfort and to get better road handling.

In the case of designing an LQR controller (called the gain matrix), one must optimal control vector must choose $u(t)$, so that the quadratic cost function is reduced. The mathematical formula of the quadratic cost function can be written as:

$$
J=\int_{0}^{\infty}\left(x(t)^{T} Q x(t)+u(t)^{T} R u(t)\right) d t
$$

Where,

$x$ and $\mathrm{u}$ are the state vector and the control vector respectively.

The designer must choose the appropriate values for both of $R$ and $Q$ to find the appropriate gain matrix using MATLAB software.

The configuration of the state variable feedback is shown in the figure 11. 


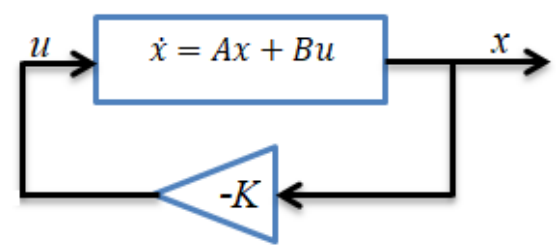

Figure 11 The configuration of the state variable feedback.

A suitable linear full-state feedback control law used as,

$u($ 回 $)=-K x($ 回 $)$

Where K represents the state feedback gain matrix for the LQR controller, which can be defined by:

$K=R^{-1} B^{T} P$

The next Algebraic Riccati Equation (ARE) is using to estimate the matrix P.

$$
A^{T} P+A P+P B R^{-1} B^{T} P+Q=0
$$

The Simulink model for the control system includes the LQR controller is shown below in Figure 12.

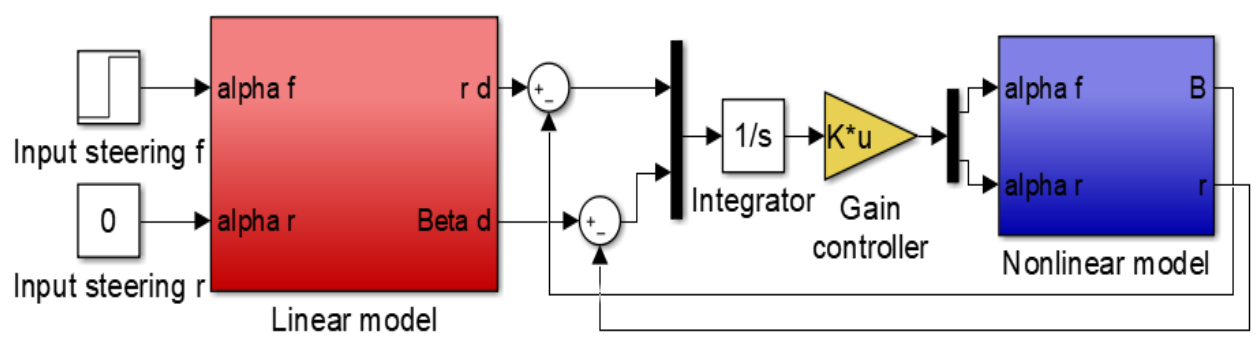

Figure $12 \mathrm{LQR}$ control system of a vehicle.

\subsection{Simulations}

In order to evaluate the performance of the developed control system, a simulation investigation is performed. The performance and dynamic behaviors of the control system with two control methods using Matlab/Simulink. We assume that the vehicle travels at a constant speed $v=20 \mathrm{~m} / \mathrm{sec}$ and the friction coefficient of the road is neglected. One driving condition is performed which is the step steering input, the steering input in the case single-track model set as a step signal with value of five degrees and set as lane change manoeuvre.

Modeling and simulations have been performed for vehicle lateral stability and different control techniques used in this work, these control techniques are Linear Quadratic Regulator (LQR) and neural network control.

Figures 13 and 14 show comparison of vehicle yaw rate performance using Linear Quadratic Regulator (LQR) and neural network control. 


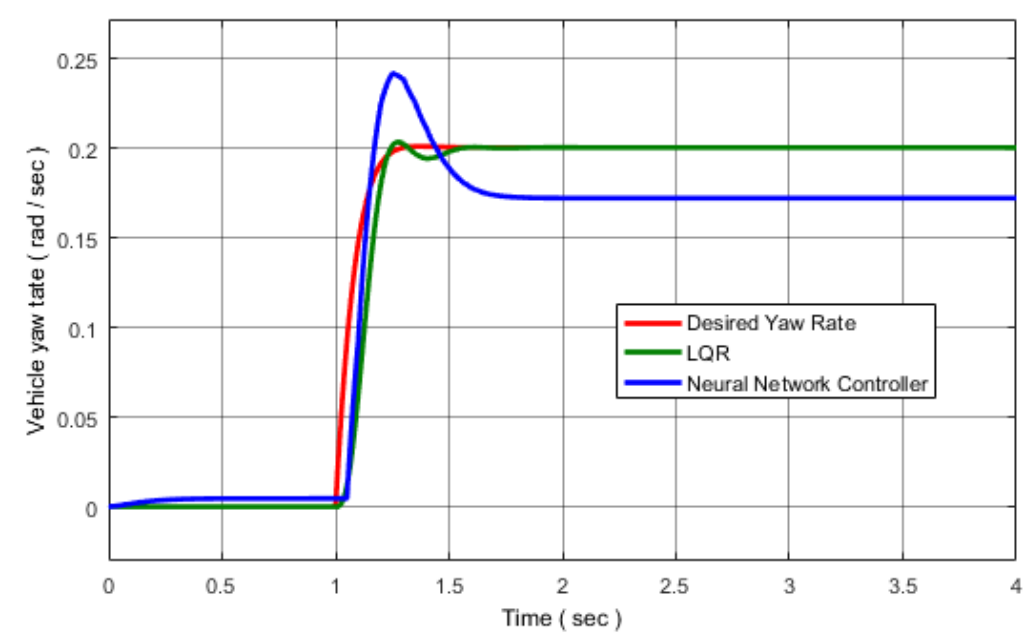

Figure 13 Vehicle yaw rate at step steering.

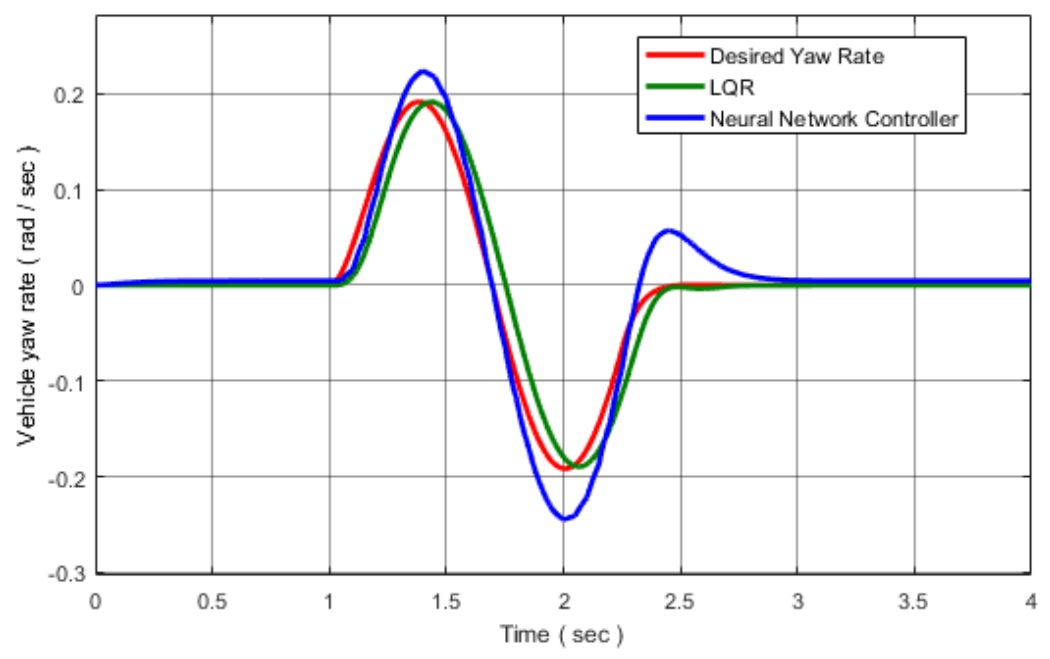

Figure 14 Vehicle yaw rate at lane change maneuver.

\section{Conclusion}

The controllers mentioned above are applied to the linear model mentioned at the beginning, we observe acceptable results that yaw rates are close to desired values. It is obvious that when the single-track model is tested, it can be seen that all of these methods have been effected the model to reach the yaw rate desired but the LQR controller is more effective than the neural network controller for yaw rate.

\section{Compliance with ethical standards}

\section{Disclosure of conflict of interest}

The authors declare no competing interests.

\section{References}

[1] M. K. Aripin, Yahaya Md Sam, Kumeresan A. Danapalasingam, Kemao Peng, N. amzah, and M. F. Ismail. A Review of Active Yaw Control System for Vehicle Handling and Stability Enhancement" International Journal of Vehicular Technology. Volume 2014, Article ID 437515, 15 pages. 
[2] M Emheisen, A Ahmed, A Emheisen and O M. Abuzaid. Evaluation Of Vehicle Stability Using Simple Single Track Model And Different Control Methods," Liceet2018 Libyan international conference on electrical engineering and technologies. 04-06/03/2018, Tripoli, Libya.

[3] Rajesh Rajamani. Vehicle Dynamics and Control. 2nd ed. Springer ScienceBusiness Media , 2012.

[4] Ogata, K. Modern Control Engineering, 5 $5^{\text {th }}$ ed. Upper Saddle River, NJ: Prentice Hall, 2010.

[5] A. A. Ahmed and A. F. Saleh Alshandoli, "Using Of Neural Network Controller And Fuzzy PID Control To Improve Electric Vehicle Stability Based On A14-DOF Model," 2020 International Conference on Electrical Engineering (ICEE), Istanbul, Turkey, 2020, pp. 1-6, doi: 10.1109/ICEE49691.2020.9249784.

[6] A. A. Ahmed and O. S. M. Jomah, "Vehicle Yaw Rate Control For Lane Change Maneuver Using Fuzzy PID Controller And Neural Network Controller," 2020 IEEE 2nd International Conference on Electronics, Control, Optimization and Computer Science (ICECOCS), Kenitra, Morocco, 2020, pp. 1-6, doi: 10.1109/ICECOCS50124.2020.9314541.

[7] A. Ali Ahmed and M. Emheisen, "Analysis of Vehicle Handling Using a Simple Track Model of Automobile," 2019 19th International Conference on Sciences and Techniques of Automatic Control and Computer Engineering (STA), Sousse, Tunisia, 2019, pp. 130-133, doi: 10.1109/STA.2019.8717244.

[8] J. Nilsson, J. Fredriksson and A. C. E. Ödblom, "Reliable Vehicle Pose Estimation Using Vision and a Single-Track Model," in IEEE Transactions on Intelligent Transportation Systems, vol. 15, no. 6, pp. 2630-2643, Dec. 2014, doi: 10.1109/TITS.2014.2322196. 